\title{
LOW-COST MEASUREMENT SYSTEM AND FILTER FOR REDUCTION OF EMC INTERFERENCES IN RADIO-FREQUENCY APPLICATIONS
}

\author{
R. Sallier ${ }^{1,2}$, L. Nietner ${ }^{1}$, U. Roland ${ }^{1,2,4}$, U. Trommler ${ }^{1,2}$, M. Kraus², F. Holzer ${ }^{2}$, C. Hoyer ${ }^{2,3}$, \\ and D. Schlayer ${ }^{4}$ \\ ${ }^{1}$ Leipzig University of Applied Sciences, Karl-Liebknecht-Str. 132, 04277 Leipzig, \\ Germany \\ ${ }^{2}$ Helmholtz Centre for Environmental Research - UFZ, Department of Environmental \\ Engineering, Permoserstr. 15, 04318 Leipzig, Germany \\ ${ }^{3}$ Otto Richter GmbH, Seelenbinderstr. 80, 12555 Berlin, Germany \\ ${ }^{4}$ Advisory Board RWTec Innovation Network, https://www.ufz.de/rwtec \\ rene.sallier@htwk-leipzig.de,lutz.nietner@htwk-leipzig.de,ulf.roland@ufz.de
}

Keywords: filter, balun, interference reduction, radio-frequency (RF) heating, prototype, electromagnetic compatibility (EMC)

\begin{abstract}
For drying building structures, dielectric heating using RF energy (13.56 MHz) is an efficient and fast alternative to conventional heating methods relying solely on heat transfer from the surface to the interior of the masonry. Another innovative application of volumetric RF heating is chemical-free pest control in wood-based materials. For such applications where the RF systems often cannot be completely shielded, the propagation or coupling of the electromagnetic waves into wires that are placed inside the field cannot be neglected which represents a certain risk. Depending on the different electromagnetic coupling mechanisms, several interference signals can be generated. In order to protect electrical equipment in the surrounding, these interferences have to be significantly reduced by electrical filters. For this purpose, a cost-effective and robust measuring concept was developed. With the help of this system, the common mode $\left(i_{\mathrm{CM}}\right)$ interference could be identified as the dominant part and the differential mode $\left(i_{\mathrm{DM}}\right)$ interference as a minor disturbance. Based on the experimental evaluation, a cost-effective filter for the respective disturbance was constructed and successfully tested.
\end{abstract}

\section{Introduction}

An innovative approach of applying radio waves (RF) for dielectric heating and drying building structures was presented in [1]. This application provides an efficient and fast alternative to conventional heating methods, which depend to a large extend on heat transfer from the heated surface into the inner volume of the material. Another innovative approach of dielectric heating via RF is the chemical-free pest control in wood-based materials [2]. Fig. 1 illustrates these two applications for the use of RF for dielectric heating. 


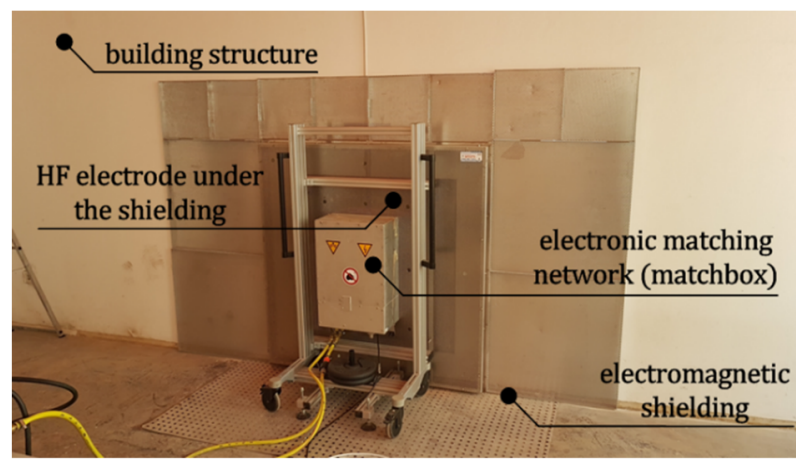

a)

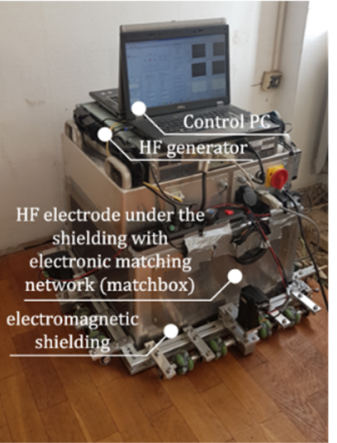

b)

Fig. 1 Electrode and matchbox systems for dielectric heating a) of building structures and

b) for chemical-free pest control in wood-based materials

In all applications of high-frequency (HF) electromagnetic waves, e.g. RF, the electromagnetic compatibility (EMC) has to be carefully considered. The undesired generation, propagation and reception of electromagnetic energy has to be prevented or suppressed as much as necessary, since detrimental effects such as electromagnetic interference (EMI) or even physical damage of equipment could occur [3]. Therefore, in Germany installations emitting RF disturbance have to be evaluated with regard to EMC for conducted interference voltages in compliance with the generic standard DIN 61000-6-3: 2011 (emission for residential, commercial and commercial areas). Since a radio-frequency of $13.56 \mathrm{MHz}$ is used in the presented applications without completely shielding, the propagation or coupling of the RF wave in existing wires in the electromagnetic field has to be examined. Depending on the different electromagnetic coupling mechanisms, different interference signals can be generated on this wire [3]. One established way in order to protect connected electrical equipment is to reduce these disturbances by means of so-called EMC filters (which are electrical filters). In order to be able to implement and use an effective electrical filter for the various types of disturbances, it is essential to identify and understand the types of coupling mechanisms and the associated disturbances. For this purpose, a simple measurement concept was developed, which works with cost-effective measuring devices in comparison to the standard expensive devices used in the HF measurement technology. On the basis of system analysis and its validation by applying a new measurement concept, cost effective filters were developed for the respective disturbance. Furthermore, the used measuring instruments are more robust compared to the conventional measuring instruments, so that they can be used in situ under field conditions.

\section{Fundamentals}

As described in Chapter I, there are different electromagnetic coupling mechanisms, which generate different disturbances in wires located in or in close vicinity of electromagnetic fields. Through a first principal functional analysis with regard to the structure of the used system, a first prediction can be made about the types of disturbance to be expected analyzing the electromagnetic coupling mechanisms. The basic structure of an RF system utilized, e.g., for drying building structures as shown in Fig. 1, is presented in Fig. 2. 


\section{High Frequency Heating}

Valencia, Spain, September 9-12, 2019

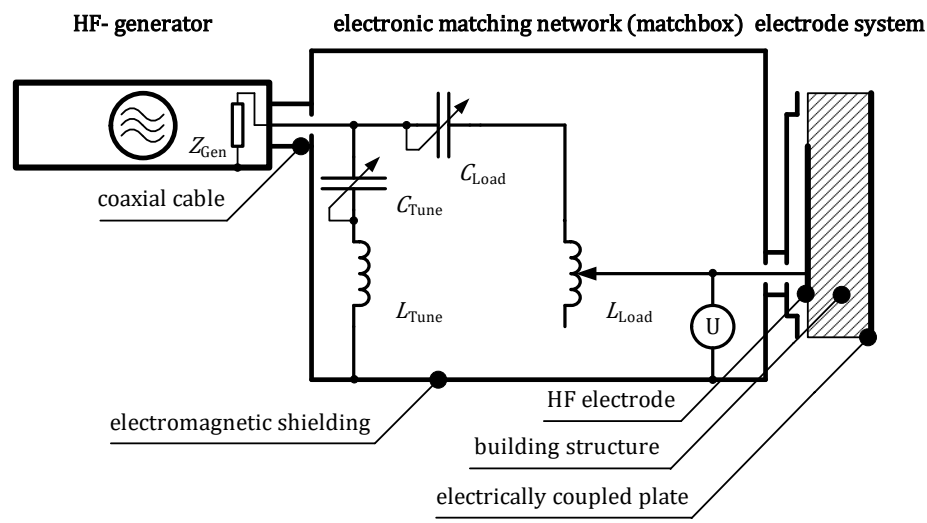

Fig. 2 Schematic representation of an RF system used for dielectric heating of building structures or chemical-free pest control

From Fig. 2, the network characteristics of the electrode system with the treated building structure acting as dielectric can be demonstrated. In more detail, the electrode system can be represented by a series connection of two capacitors (Fig. 3 a)) and, thus, it is possible to describe the total capacitance by using a single capacitor (Fig. 5 a)). When a wire (two- or three-wire leads) is located between the electrodes or in the vicinity of the electrode system, disturbances are coupled into this wire in accordance with the mentioned coupling mechanisms. The occurring coupling mechanisms are the capacitive - by E field (Fig. $3 \mathrm{~b}$ ) and Fig. 4 b)) and the inductive coupling $-\mathrm{H}$ field (Fig. 3.b)) correlated with the arrangement of the electrodes systems vs. wire leads.

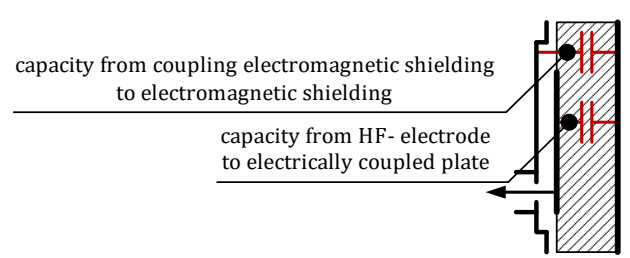

a)

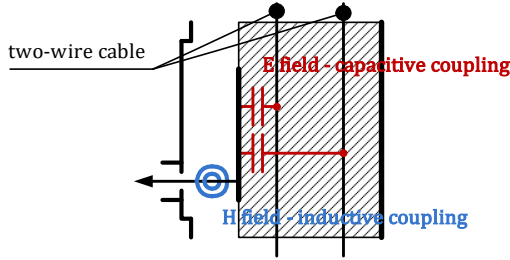

b)

Fig. 3 a) RF Electrode system with building structure as dielectric and resulting capacities, b) acting coupling mechanisms 


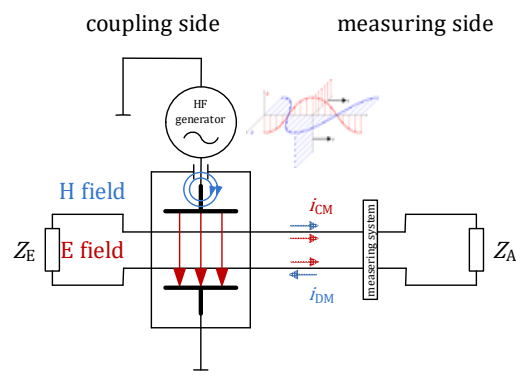

a)

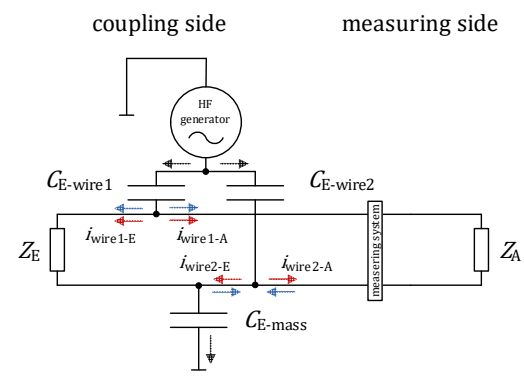

b)

Fig. 4 a) Simplified schematic representation of the coupling mechanisms in a two- or three-wire lead system, b) simplified scheme of the E field coupling paths

As already described, the whole system of the series connection of two capacitors can be transferred into a system consisting of only one capacitor which is installed between the RF voltage side and the ground (see Fig. 4 a)). $Z_{\mathrm{E}}$ and $Z_{\mathrm{A}}$ are the input and output impedances, respectively, of the lead wires in the electromagnetic field. The two dominant coupling mechanisms (via E field and $\mathrm{H}$ field) result primarily from the arrangement of the electrodes relative to the wires. In order to identify the contributions of the two disturbances due to the different coupling mechanisms, it is crucial to detect the disturbances also separately employing an appropriate measurement (see Fig. 5 a)). The coupling mechanisms can be fundamentally described using Maxwell's equations [4, 5].

\section{Measurement system}

To determine the respective disturbance currents for common mode $\left(i_{\mathrm{CM}}\right)$ and differential mode $\left(i_{\mathrm{DM}}\right)$, a measuring system consisting of three current clamps was constructed with the respective consideration of the current directions of the clamps (Fig. 5). The measurement of these disturbance currents with current clamps is possible without significant influences on the system to be measured since the measurement principle is based on inductive coupling. The system which was used for RF heating is a $5 \mathrm{~kW}$ system from Trumpf Hüttinger (Freiburg/Brsg., Germany). The measurements were carried out for a power range between 0 and $500 \mathrm{~W}$.

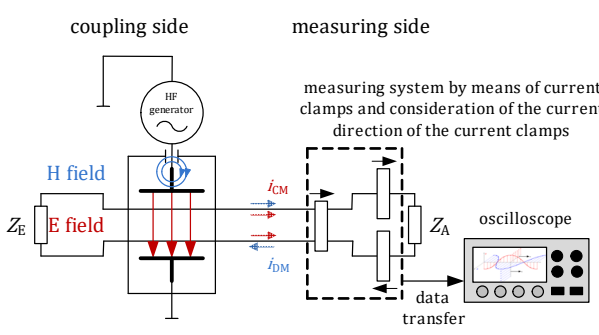

a)

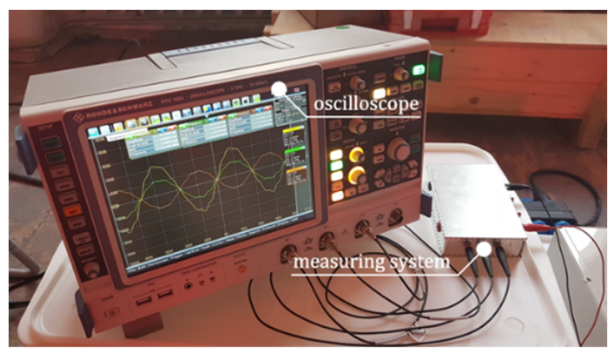

b)

Fig. 5 a) Arrangement for measuring the common mode and differential mode currents in a two- or three-wire lead system exposed to electromagnetic fields, b) measuring system 
The integral disturbance currents were measured with three Tektronix current measuring probes (CT1) in combination with a Rohde \& Schwarz oscilloscope (RTO 1024, probe input $>10 \mathrm{M} \Omega$ ). The disturbance currents $i_{\mathrm{CM}}$ and $i_{\mathrm{DM}}$ were determined from the measured values applying the following equations:

and

$$
i_{\text {wirel }}=i_{\mathrm{CM}}+i_{\mathrm{DM}}
$$

and

$$
i_{\mathrm{wire} 2}=i_{\mathrm{CM}}-i_{\mathrm{DM}}
$$

$$
i_{\text {wire } 12}=2 i_{\mathrm{CM}} \text {. }
$$

The disturbance currents $i_{\mathrm{CM}}$ and $i_{\mathrm{DM}}$ can already be determined from the first two equations. The third equation is used to validate the $i_{\mathrm{CM}}$ result derived from the first two equations. For this purpose, the forward wire (wire1) and return wire (wire2) of a two-wire lead were routed through a common clamp meter (green graph in Fig. 6). Thus, the components of $i_{\mathrm{CM}}$ of the lead in the current clamp add up and the components of $i_{\mathrm{DM}}$ were compensated due to opposite signs. With the help of the other two current clamps, the currents in the forward $\left(i_{\text {wire } 1}\right)$ and return $\left(i_{\text {wire2 }}\right)$ wires were determined. The disturbance currents calculated from the equations are represented by the red and blue curves in Fig. 6. Both measurement options of $i_{\mathrm{CM}}$ show, within the measurement accuracy, a very good agreement as also shown in Fig. 6 by the green and the red curves.

This diagram exemplarily shows the resulting disturbance currents for the application illustrated by Fig. 1 a. Analyzing these measurements, it can be clearly seen that the dominant mode of disturbance in this case is common mode (CM). Thus, the measurement of disturbance currents supports the results of the system analysis given in Chapter II. From Fig. 6, it has to be also concluded that the disturbance currents in the wires due to an exposure to an electromagnetic field can not be ignored. Of course, the position of the wire with respect to the electrode system plays a significant role for the amplitude of the disturbance currents as well as for the correlation with the generator output voltage. At a certain level of disturbance currents, the communication within the electrical equipment may be disturbed or even electrical devices could be affected. Thus, there is a need to analyze, control and, if necessary, to reduce these disturbance currents.

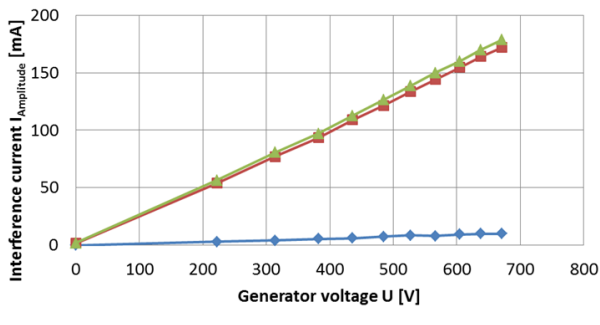

$\rightarrow$ I_DM $\rightarrow$ - I_CM - I_CM from common mode measurement

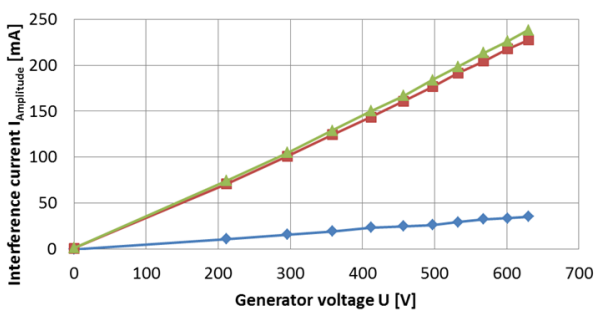

$\rightarrow 1 \mathrm{DM} \rightarrow-\mathrm{C} C \mathrm{CM} \rightarrow \mathrm{I}$ CM from common mode measurement

Fig. 6 Identification of common (CM) and differential mode (DM) interferences without filter and two different measuring modes used for common interference: wire directly under the electrode (left) and wire beside the electrode (distance about $1.5 \mathrm{~m}$ from the RF electrode, right) 


\section{Filter}

On the basis of the disturbance current analysis in Chapter II and the results obtained for an application described in Chapter III showing a dominance of CM over DM conventional filter concepts such as, e.g., suction circuits are less suitable or even not effective at all [6]. This is due to the lack of potential difference between both wires. Thus, a new filter concept must be employed for this type of disturbance current. A well-known approach in the world of radio amateurs is the so-called balun (balanced-unbalanced, Fig. 7 and [7]). Such baluns are used in high-frequency technology mainly as balancing unit. Baluns consist of electrically poor or non-conductive ferrimagnetic materials as core material, e.g. 4W620. For each type of disturbance (CM oder DM), a filter effect can be realized with a corresponding coil arrangement. In Fig. 7, the winding directions are shown for a CM and DM lock, respectively.

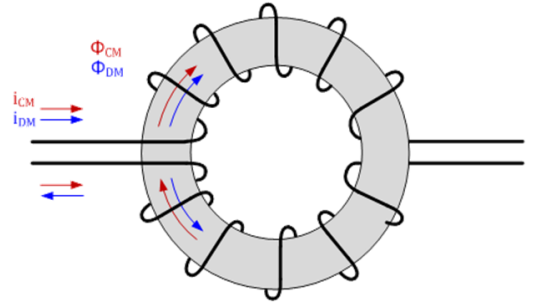

a)

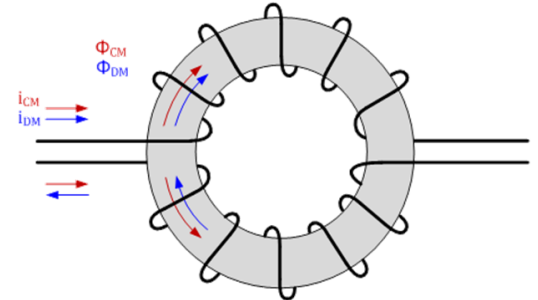

b)

Fig. 7 a) CM lock and b) DM lock

The filter effect of a balun [7,8] is based on the well-known principle of induction. When a periodic current flows through a wire, a periodic magnetic field is formed around this wire. According to Ampère's law, the current $I$ is calculated as a ring integral over the magnetic field strength $H$ with the path $s$ leading to the induction voltage $\Theta$. By winding the wire into a coil, $\Theta$ can be multiplied. The voltage $\Theta$ generates a magnetic flux $\Phi$ that is also dependent on the magnetic resistances in the considered circle. The current direction of the coil determines the direction of $\Phi$. Thus, a periodic magnetic flux is generated in the ferrite material depending on the winding direction and the direction of the periodic disturbance current. For coils with ring cores and without air gap, the inductance $L$ can be determined, according to [9], by the following equation:

$$
L=\frac{1}{2 \pi} \cdot \mu_{0} \cdot \mu_{i} \cdot N^{2} \cdot h \cdot \ln \left(\frac{d_{a}}{d_{i}}\right)
$$

It can be simplified by introducting the geometry parameter $A_{\mathrm{L}}$ leading to

$$
L=A_{L} \cdot N^{2} \text {. }
$$

Here, $N$ is the number of coil turns, $h$ the length of the core, $d_{\mathrm{a}}$ and $d_{\mathrm{i}}$ are the outer and inner diameters, respectively, $\mu_{0}$ is the magnetic field constant $\left(4 \pi 10^{-7} \mathrm{Vs} \mathrm{A}^{-1} \mathrm{~m}^{-1}\right)$ and $\mu_{\mathrm{i}}$ as relative permeability of the material. Based on these theoretical principles and applying the 4W620 ferrite material after several tests with the impedance value from the datasheet of the provider $\left(\mu_{\mathrm{i}}=620\right)$, two baluns were realized. The baluns, one for reducing the $\mathrm{CM}$ 
disturbance (CM lock) and one for reducing the DM disturbance (DM lock), were installed in one chassis (see Fig. 9 a)).

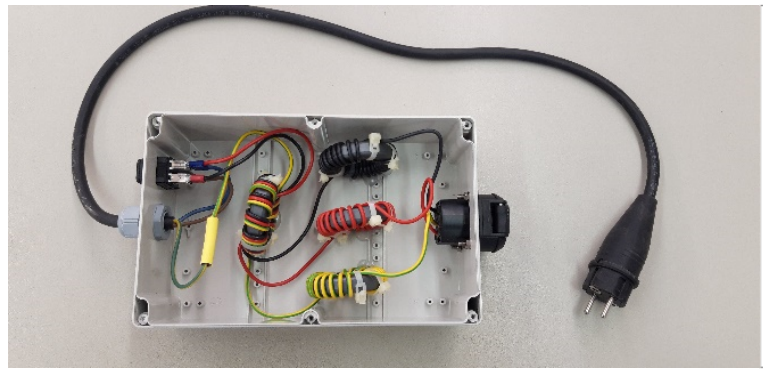

a)

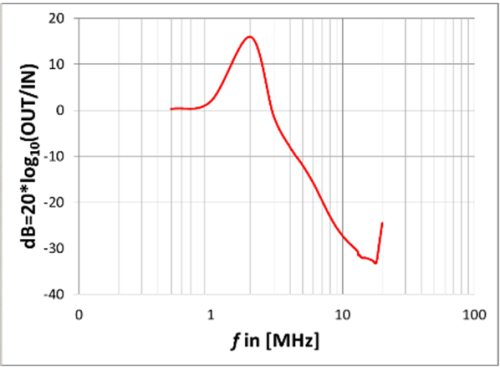

b)

Fig. 9 a) Realized filter consisting of a CM lock and a DM lock, b) transfer function of the total balun, measured between phase and ground wires

Measurements of $i_{\mathrm{CM}}$ and $i_{\mathrm{DM}}$ using the realized filter (Fig. 9 a)) showed a good matching result within the measurement accuracy as can be seen from Fig. 10 (green and red graphs for $i_{\mathrm{CM}}$ and blue graph for $i_{\mathrm{DM}}$ ). But, more important, the graphs in Fig. 10 show the resulting markedly lower disturbance currents after using the new filter exemplarily for the application of drying brickwork by RF heating [1] presented in Fig. 1 a).
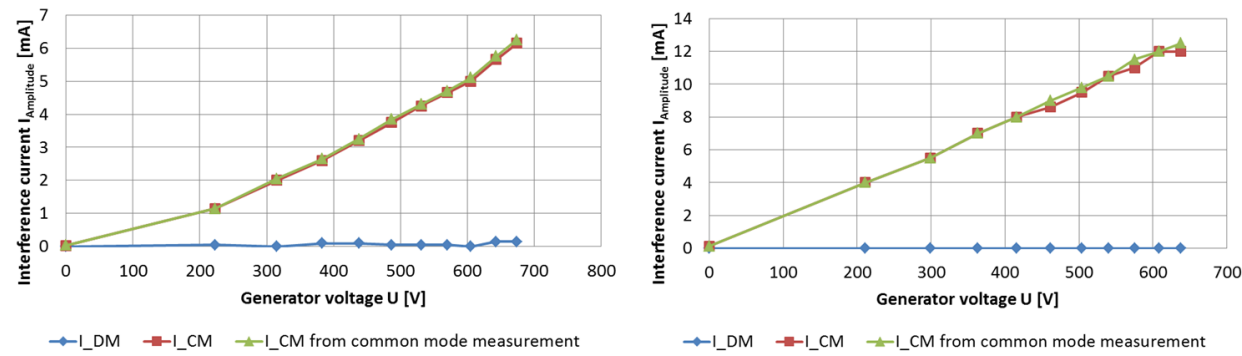

Fig. 10 Identification of CM and DM interferences with the new filter determined by applying two different measuring modes for common interference: wire directly under the electrode (left) and b) wire beside the electrode (right)

From a comparison of the disturbance current amplitudes presented in Fig. 6 and Fig. 10, it can be concluded that the realized integral filter has a marked filter effect with an average

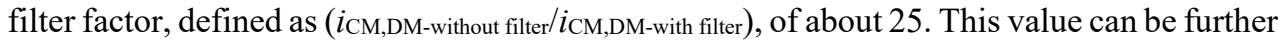
enhanced using the developed principle thus providing an important tool for RF applications in the future.

\section{CONCLUSION}

The reduction of electromagnetic noise is an important aspect for ensuring electromagnetic compatibility (EMC) for RF methods in many application fields. It is especially crucial for situations where complete surrounding of the electrodes and the treated dielectric by an electromagnetic shielding (Faraday cage) is not possible. Often, this corresponds to 
processes with large application potential for RF heating such as drying of brickwork and building structures or chemical-free pest control, e.g. of parquet floor.

In order to develop appropriate solutions for EMC, typical RF heating systems were carefully analyzed with respect to the emission of electromagnetic waves. The main coupling mechanisms were identified which are most relevant for situations where electrical wires cross the treatment or are installed in the vicinity of the electrode system.

Based on detailed investigation of common and differential mode disturbances, the common mode was identified as the more critical influence. For doing so, appropriate measuring regimes were developed and applied, exemplarily for RF drying applications. Thus, a robust and cost-effective measuring system based on current clamps was developed and successfully tested for the in situ detection of RF interferences and identification of coupling mechanisms.

An important conclusion from the measurements is the necessity of developing suitable filter systems for the RF applications. Therefore, based on the concept of baluns, a filter was developed which reduced the coupling disturbance current by a factor of approximately 25 . Further developments can enhance this parameter. In practise, this filter reduced coupling disturbance to an extent that downstream devices (e.g. computers and temperature monitors) were protected against possible disturbance and the communication between the devices at the site was not endangered.

\section{References}

1. Roland, U., F. Holzer et al., COMPEL, 2018, 37, 1933-1942

2. Hoyer, C., C. Pfütze et al., Chem. Eng. Technol., 2018, 41, 108-115

3. Nedtwig, J., M. Lutz, „WEKA Praxishandbuch Elektromagnetische Verträglichkeit“", Berlin, 1999, ISBN 3-8111-7890-3

4. Schwab, A.J., „Elektromagnetische Verträglichkeit“, Springer Verlag, Heidelberg, 1996

5. Morgan, D., „A Handbook for EMC Testing and Measurement“, IEE Electrical Measurement Series 8, 1996

6. Chen, W.-K., „The Circuits and Filters Handbook“, CRC Press, IEEE Pres., 1995, ISBN 0.8493-8341-2

7. Ellis, G., „Electronic Filter Analysis and Synthesis” Artech House, 1994, ISBN 089006-616-7

8. Tietze, U., C. Schenk, E. Gramm, „Halbleiter Schaltungstechnik“, Springer Verlag, Heidelberg, 2016, ISBN 978-3-662-48354-1

9. Schindler, G., Presentation „Magnetische Bauteile und Baugruppen“, attempo, Rottenburg, Version 1.1, 25.06.2010 